\title{
A Novel Cascading Failure Model on City Transit Network
}

\author{
Tao $\mathrm{He}^{1, \mathrm{a}}$, Ning Zhu ${ }^{2, \mathrm{~b}}$, Zhenshan $\mathrm{Hou}^{3, \mathrm{c}}$ and Guixi Xiong ${ }^{4, \mathrm{~d}}$ \\ ${ }^{1,2,3,4}$ School of Computer Science and Engineering, Beihang University, Beijing, China \\ ahtwalnut@buaa.edu.cn, ${ }^{\text {b } z h u n i n g @ b u a a . e d u . c n, ~}{ }^{c}$ housir@buaa.edu.cn, ${ }^{\text {d }}$ guixixiong@buaa.edu.cn
}

Keywords: cascading failure; path navigation strategy; city transport network; capacity-load model;

\begin{abstract}
Urban public transportation network is a typically complex network and the local fault of network often leads to serious systemic impact, causing cascading failure. Research on cascading failure of the bus network, is advantageous to understand of the potential key individuals of the network, so as to guide the rational planning of transit network. At first, this paper proposed the path navigation strategy based on the transfer bus to describe the flow propagation law of transit network, and after which an improved failure model of the bus network based on capacity-load model was put forward. Finally, the experimental analysis was conducted based on real traffic data of Beijing, and under the improved path navigation strategy, the correlation between node load and real data reached $99.61 \%$, accord with the real law more than the traditional path navigation strategy. Cascading failure simulation illustrated that even small-scale attacks could lead to systemic paralysis, causing serious impacts on the structure and function of network, meanwhile the damage to the functional integrity is more severe than structural integrity. Conclusion is conducted that results can guide the bus lines and citizens' travel.
\end{abstract}

\section{Introduction}

Cascading failure [1] is that when some nodes or edges in the network malfunction due to interference, the failure of a large scale of nodes or edges in the network will occur through a coupling relationship with the surrounding nodes or edges. At present in the field of transportation, research on cascading failure model is mainly based on the load capacity model. Initially, the nodes or edges in the network are endowed with certain load and capacity. Then some nodes and edges of the network are attacked and become failure. Through the navigation strategy, the load is redistributed to the network. The load of the nodes or the edges exceeds their capacity, so the cascading failure is caused. Wu, Gao, Sun [2] [3,4] made a large number of analysis on the cascading failure phenomena of the traffic network and found that big nodes or high-loaded nodes were likely to cause the collapse of the entire network. Wang Zhengwu, Kuang Aiwu, Wang Hejie [5] put forward a cascading failure model of traffic network based on double network and proposed a method for calculating the importance of nodes. The results showed that the structure of traffic network and the behavior of people had an important influence on the importance pf nodes. In addition, the Motter [6, 7] presented a cascading failure model based on the betweenness, calculating the load of network nodes through the shortest path and analyzing the cascading failure of the network. Due to the limitations of the shortest path navigation strategy, Wei Wang [8] put forwarded a cascading failure model based on random walk betweenness. However, these studies are lack of validation of the propagation law of real passenger flow and the use of the navigation strategy is lack of consideration of the limitations of line design in the real network.

\section{Transfer-based path navigation strategy}

Transfer-based path navigation strategy mainly aims to afford an optimized bus lines from passengers' originate to his destination, which is usually simplified with OD, surely including bus stops needed to transfer. The main flow processes include two parts:

Establishing an optional bus line set for the path. We use $p$ to represent the node of the bus network, especially transfer node which is the intersecting node of at least two different bus lines, $e$ to 
represent the edge of bus network and $l$ to represent the bus line. We also need to know the relationship between node $p_{i}$ and node $p_{j}$, so we propose indicator function $a_{i j}$. If the two nodes connected directly, $a_{i j}=1$ else $a_{i j}=0$. Accordingly, $\operatorname{dist}\left(p_{i}, p_{j}\right)$ is defined as the distance formula of two points on the earth surface.

As known to us all, path from $p_{i}$ to $p_{j}$ is not only one but many, so we use a path set, $\operatorname{PATH}\left(p_{i}\right.$, $\left.p_{j}\right)=\left\{\right.$ path $_{i j} 1, \ldots \ldots$, path $\left._{i j} n\right\}$ to represent. In this set, path ${ }_{i j} k$ means the $\mathrm{k}^{\text {th }}$ path from $p_{i}$ to $p_{j}$.

For every element $e_{a b}$ in $p a t h_{i j}=\left\{e_{i 1}, \ldots . ., e_{a b}, \ldots \ldots, e_{n j}\right\}$, it means node $p_{a}$ and $p_{b}$ is connected directly, and the physical distance is $\operatorname{dist}\left(p_{a}, p_{b}\right)$. Therefore, the physical distance of $p a t h_{i j}$ is sum of the length of all adjacent points on the path, computed as follows:

$$
\operatorname{len}\left(\text { path }_{i j}\right)=\sum_{\text {eab } \in \text { pathij }} \operatorname{dist}\left(p_{a}, p_{b}\right)
$$

For adjacent two elements, $l_{c}$ and $l_{d}$ in path $_{i j}=\left\{l_{1}, \ldots . ., l_{c}, l_{d}, \ldots \ldots, l_{n}\right\}$, the order of every element is fixed, it must meets: to the transfer node $p_{i}$ in the bus network, $p_{i} \in l_{c}$ and $p_{i} \in l_{d}$. What's more, the sequence of transfer nodes in path decides the sequence of bus lines.

The minimum cost of chosen path. Considering transfer distance ignored in traditional path navigation strategy, this paper propose the function of transfer number for the path as follows:

$$
\operatorname{tr}\left(\operatorname{path}_{i j}, n\right)=\left\{\begin{array}{l}
0, n=1 \\
\operatorname{tr}\left(\text { path }_{i j}, n-1\right), \ln _{\mathrm{n}-1}=\mathrm{ln}_{\mathrm{n}} \\
\operatorname{tr}\left(\text { path }_{i j}, n-1\right)+1, \mathrm{ln}_{\mathrm{n}-1} \neq \mathrm{ln}_{\mathrm{n}}
\end{array}\right\}
$$

For a path consisting of same bus line sequence, there will be different transfer ways. Least transfer number is the best choice, symbolized with Ltr(pathij).

$\operatorname{cost}\left(\right.$ path $\left._{i j}\right)$ is defined as the total cost of the path , as follows:

$$
\cos t\left(\text { path }_{i j}\right)=\alpha \times \beta \times \operatorname{Ltr}\left(\text { path }_{i j}\right)+(1-\alpha) \times \operatorname{len}\left(\text { path }_{i j}\right)
$$

Cost mainly comes from two concepts, one cost in transfer and the other in physical distance. We use $\alpha$ to represent the weight factor between the physical distance and the transfer cost. To simplify calculation, transfer cost is uniformly expressed with $\beta$.

Assign $\alpha=0.99$ and as a result of $\alpha>>(1-\alpha)$, this strategy is to select the shortest path in the case of the least transfer number, which reflects the consideration of the passengers' travel activity due to the limited accessibility of the public transport network.

\section{Cascading failure model}

When a bus stop load more than its capacity called node attacked, we proposed a novel cascading failure model based on the transfer-based path navigation strategy. At the same time, a functional indicator is put forward to calculate the ratio of effective OD flow as the function of the net is to deliver the OD flow.

The failure can be observed in Fig. 1, the network in the model description graph has five bus lines and nine bus stops and the process happens as follows:

Step1: Node $P_{3}$ is attacked, marked with failed;

Step2: edges between $\left(P_{1}, P_{3}\right),\left(P_{2}, P_{3}\right),\left(P_{3}, P_{4}\right)$ and $\left(P_{4}, P_{6}\right)$ failed in success, so $l_{1}, l_{4}$ have to find new paths to connect the broken parts. As $l_{4}$ cannot find the path from $P_{1}$ to $P_{6}$, it is cut into two lines, $l_{4}$ and $l_{4}$ ', which respectively only own half of previous capability. And $l_{1}$ remains unchanged as it finds a new path $P_{2^{-}}>P_{5^{-}}>P_{6^{-}}>P_{7-}>P_{4}$ for $P_{2}$ to $P_{4}$.

Step3: calculating the new capacity for each node and reallocate the OD flow;

Step4: $P_{5}$ and $P_{7}$ fail because their load is more than their capacity. 


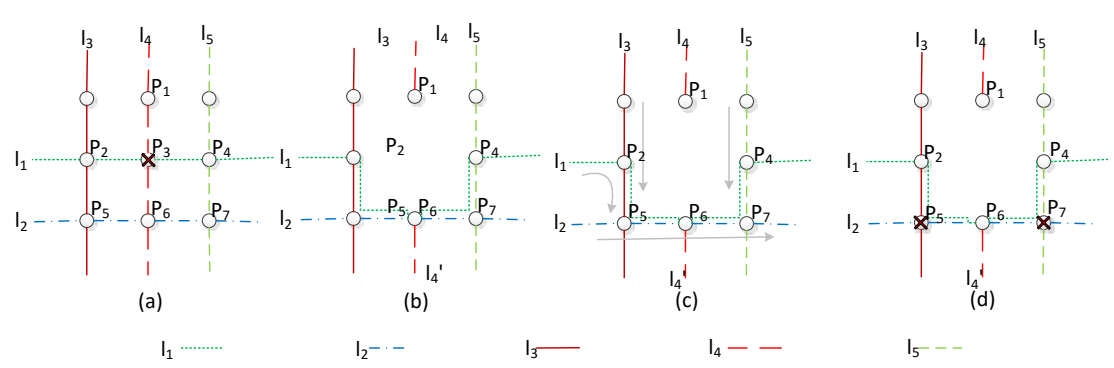

Fig. 1 Example of the cascading failure.

Based on the above points, the cascading failure model is described as follows: load.

Initial Status: Distribute the capacity for each line so the node capacity is enough to hold the initial

Attack Status: Delete the nodes which carry most load.

Update Status: Find the new paths in the broken lines to keep line-integrity, or cut the line and update the capacity if the path does not exist.

Flow allocate: Calculate the path of each od pair according to the transfer-based navigation policy and update the node load.

Fail judgement: If all the node load is within the capacity, then stop the process, otherwise go to next step.

Update the network: Delete the nodes whose load is over the capacity.

Effect evaluate: Calculate the indicator Ep to denote the structural integrity which equals the ratio of the effective nodes, and Eod to the functional integrity which equals the ratio of the effective od flow.

\section{The experimental simulation}

This paper uses the real traffic data in Beijing to make the experimental analysis, including data of bus lines and passenger flow in the card. Bus data contains information of 1598 bus lines and 6119 bus stations in Beijing. Data in the card recorded a total of 43919968 public bus travel records from 2015.01.04 to 2015.01.10. In this part, the navigation strategy and cascading failure model of this paper will be verified and analyzed by using this real data.

Verification of Path Navigation Strategy Based on Transfer. In this part, the traffic load LoadS of each node calculated from transfer-based shortest path strategy and traditional shortest path algorithm will be compared with the real passenger load LoadR respectively. The real traffic flow distribution is calculated by the line number corresponding path in the data record of the card. The comparison results are shown in Fig. 2.
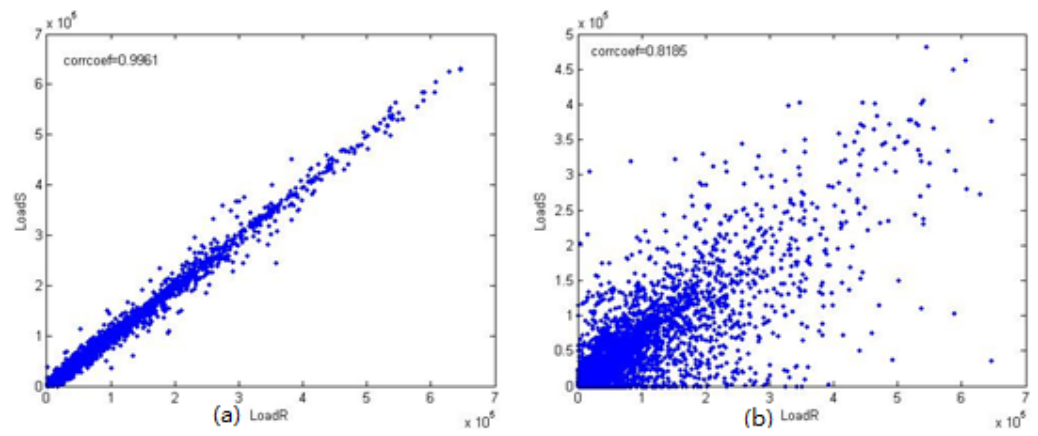

Fig. 2 A comparison chart of the load of nodes in the Transfer-based path (a) and Shortest distance path (b) navigation policy

In the Fig. 2, the horizontal axis represents the real traffic load of nodes and the vertical axis represents the simulative traffic load of nodes. In the upper left corner, Pearson coefficient between the load LoadS of nodes in the corresponding strategy and the real load LoadR of node is recorded. 
Through the comparison, it can be found that the distribution of the scattered points in (a) is more concentrated and the fit with the load of real nodes is higher than it in the (b) .

In the figure of distribution of scattered points, the density of scatter points in the lower left corner is greater than them in the upper right corner. It is proved that the number of low-load nodes is greater than the number of high-load nodes in the network and the bus network is unbalanced as a complex network in the load of nodes.

Analysis of Cascading Failure Model of Bus Network. When the bus network is attacked, how the integrity of network function structure changes with the number of nodes pnum in the initial attack and index of saturation $\gamma$. Two indicators reflect this change: the structural integrity Ep: the ratio of the number of effective nodes in the network to the original number of nodes in the network; functional integrity Eod: the ratio of effective travel demand to the original travel demand. The attack is based on node load to make node failure. The change trend of network integrity with attack scale and saturation index is compared and analyzed.

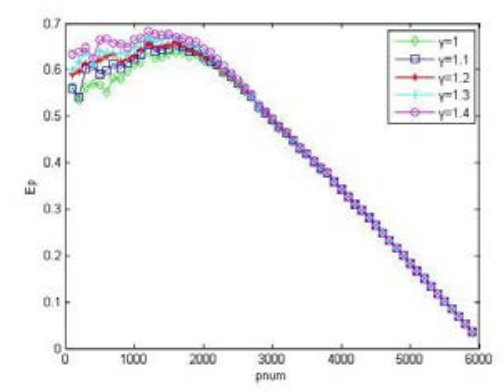

(a)

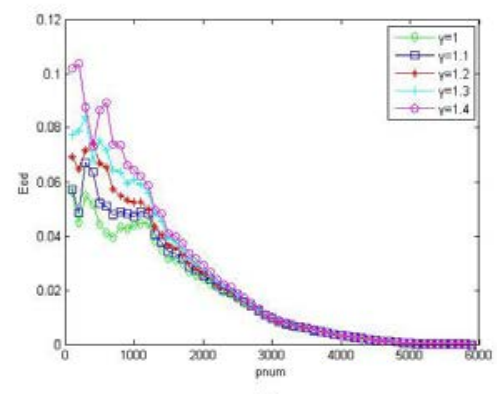

(b)

Fig. 3 Structural integrity (a) and Functional integrity (b) with different initial attacking number. The capacity coefficient are set to $1,1.1,1.2,1.3,1.4$ respectively.

Fig. 3 show the change of network integrity with attack scale. It can be known that the overall trend of the network integrity is declining with the increase of attack scale. However, when the initial attack scale is less than 1500, there is a rising trend. Because when part of the key nodes in the network is attacked initially, the surrounding larger load of the nodes also received the attack, leading to the inhibition of cascading failure in some degree. In addition, it can be found that in the same situation the functional integrity of the network is less than 0.1 , which is more destructive than the structural integrity. It means that the nodes that play an important role in the network all have failed. Especially when pnum is around 2000, the network structure maintain the integrity of the 0.5, but the functional integrity Eod has been close to 0.02, which means there is no traffic on the network basically.

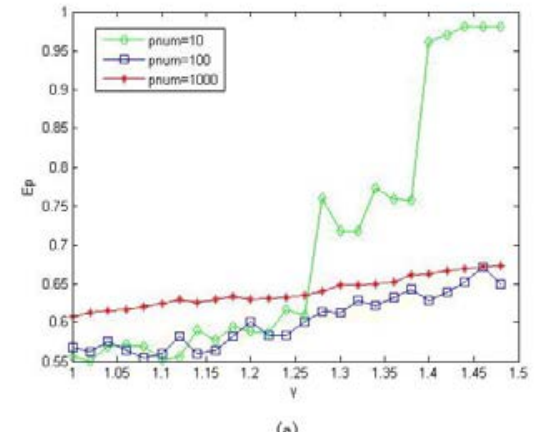

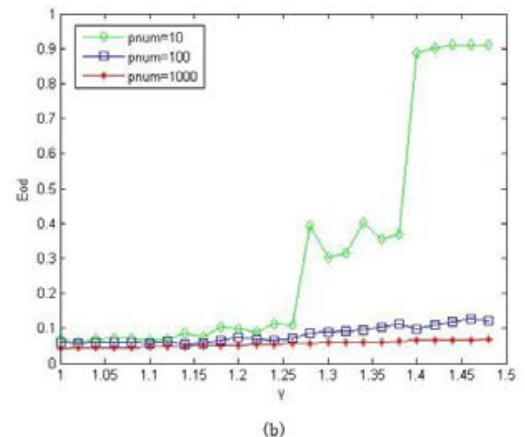

(b)

Fig. 4 Structural integrity(a) and Functional integrity(b) with different capacity coefficient. The initial attacking number are set to 10, 100, 1000 respectively. 
It can be seen in Fig. 4 that as $\gamma$ grows, both Ef and Eod increase, with Ef more obviously. Especially when $\gamma$ exceeds 1.4 , the structural and functional integrity improve rapidly. That means by amplifying the capacity of the net, the cascade can be prevent when initial damage is small. However, the function improves little when the initial damage raises to 100, as the key nodes mainly fail.

\section{Summary}

The stability is not only important to the travel activity, but also the production and service of the city. Focusing on the cascading model of urban bus network, this paper firstly proposed a transfer-based navigation strategy, and improved the cascading model based on the 'capacity-load' model. The experiments showed the navigation strategy is reliable to describe the real rule of the OD flow propagation on the bus net. And the cascading model illustrated that the function of the net is more fragile than the structure. This work is meaningful to conduct the prevention measure and transport planning.

\section{Acknowledgements}

This research derived from the Project whose name is City multi-mode data system interconnect technology and support environment (2013AA01A601), and the project is supported by the National High Technology Research and Development Program (863 Program), which attached to Smart Cities (Phase II).

\section{References}

[1] Crucitti P, Latora V, Marchiori M. Model for cascading failures in complex networks[J]. Physical Review E, 2004, 69(4): 045104.

[2] Gao Ziyou,Sun Huijun, Zhao Hui. city traffic system complexity [M]. Science Press, 2010

[3] Wu J J, Gao Z Y, Sun H J. Effects of the cascading failures on scale-free traffic networks[J]. Physica A: Statistical Mechanics and its Applications, 2007, 378(2): 505-511.

[4] $\mathrm{Wu} J$ J, Sun H J, Gao Z Y. Cascading failures on weighted urban traffic equilibrium networks[J]. Physica A: Statistical Mechanics and its Applications, 2007, 386(1): 407-413.

[5] Wang Zhengwu, Kuang Aiwu, Wang Hejie. The importance of considering cascading failure of traffic network nodes [J]. Highway traffic technology, 29, 2012 (5): 96-101.

[6] Motter A E, Lai Y C. Cascade-based attacks on complex networks[J]. Physical Review E, 2002, 66(6): 065102.

[7] Motter A E. Cascade control and defense in complex networks[J]. Physical Review Letters, 2004, 93(9): 098701.

[8] Wang Wei ,Di Peng, , Hu Bin. Cascading failure model based on random walk betweenness [J]. systems engineering and electronics, 2012, 34 (9): 1914-1917.

[9] Shen Di, Li Jianhua, Xiong Jinshi, et al. A cascade failure model [J]. Complex system and complexity science, 2014, 11 (3): 12-18.

[10]Chen S, Huang W, Cattani C, et al. Traffic dynamics on complex networks: a survey[J]. Mathematical Problems in Engineering, 2011, 2012

[11]Bernardo M, Manfredi S, Garofalo F, et al. Load distribution in small world networks[R]. 2005. 